\title{
Dosing Days to Detection
}

National Cancer Institute

\section{Source}

National Cancer Institute. Dosing Days to Detection. NCI Thesaurus. Code C117041.

The number of days from the start of dosing to the earliest detection of a condition or pathogen. 